\title{
RECONSTRUYENDO LA MEMORIA COLECTIVA DE LOS CUIDADOS PARA LA SALUD EN EL VALLE DEL RÍO CIMITARRA: UNA PROPUESTA INVESTIGATIVA
}

\author{
RESTORING THE COLLECTIVE MEMORY OF HEALTH CARES IN THE \\ VALLEY OF THE CIMITARRA RIVER: A RESEARCH PROPOSAL
}

\author{
Celmira Laza VásqueZ*
}

\begin{abstract}
RESUMEN
Se presenta la experiencia de una propuesta investigativa a mediano plazo que se desarrolla en la actualidad. En ésta se pretende comprender los cuidados de la salud en el contexto de las dinámicas culturales, sociales, económicas y políticas que los han determinado para aportar a la construcción de los procesos de empoderamiento de la salud y consolidación de la identidad de una población rural que en su conformación han influido diferentes acervos culturales, y condiciones sociopolíticas y económicas producto del desplazamiento forzado por la violencia y el conflicto social y armado que se desarrolla en la región del Valle del río Cimitarra - Magdalena Medio colombiano. Se aborda el contexto investigativo y la "ruta" que se sigue: la construcción de la experiencia de los problemas de salud, descripción de las prácticas de cuidado, de los agentes tradicionales, los recursos tradicionales utilizados para el cuidado y el gasto en salud de las comunidades. Se plantea una triangulación por la complejidad del fenómeno de estudio. Los referentes teóricos utilizados son la Teoría de la universalidad y diversidad de los cuidados culturales, la Sociología médica y la Antropología médica crítica. Metodológicamente, se asume el enfoque cualitativo y para la recolección de la información, técnicas de la etnografía y la investigación-acción. El método interpretativo-hermenéutico se propone para el análisis de la información.
\end{abstract}

Palabras clave: Cuidado para la salud, cultura, reconstrucción, memoria colectiva, salud, enfermedad.

\begin{abstract}
The experience of a research proposal of medium term that is currently developing is presented. The purpose, is trying to know the health care in the dynamic, social, economical and political context in which they have determined them in order to contribute to the construction of the health empowering and consideration of the identity of rural population, that in its conformation, different cultural backgrounds and socio-political and economic conditions, product of forced relocation produced by violence have influenced them, and the social and armed conflict that is been developed in the Valley of the Cimitarra River - Colombian Medium Magdalena. The research context is approached and the "route" following is: the construction of the health problems experience, description of the care practices, the traditional agents, traditional resources used for care and the communities' health expenses. An attempt of triangulation by the complexity of the study phenomenon is set out. The theoretical referents used are the Universality Theory and the diversity of the cultural care, the Medical Sociology and the Critical Medical Anthropology. Methodologically, the quantitative approach is assumed, and for the collection of information, ethnography techniques and action-research. The interpretative-hermeneutic method is proposed for the analysis of the information.
\end{abstract}

Key words: Health care, culture, restoring, collective memory, health, illness.

Fecha recepción: 17/10/08 Fecha aceptación: 26/06/09

*Enfermera. Magíster en Enfermería con énfasis en cuidado de la salud materno perinatal-Universidad Nacional de Colombia- Bogotá. Fundación Universitaria del Ârea Andina. Bogotá-Colombia. Dirección: Calle 71 №. 13-21. Bogotá, Colombia. Email: claza@areandina.edu.co celmira.laza@gmail.com 


\section{INTRODUCCIÓN}

Intentar reconstruir la memoria colectiva en torno a los cuidados para la salud de un pueblo significa un ejercicio investigativo, que además de ser a mediano o largo plazo, implica más que un acercamiento, una inmersión en la vida cotidiana de éste, en su pasado y su proyección hacia el futuro. Esto, pensado en un quehacer investigativo arduo y paciente, pero a la vez respetuoso de la voz del otro. Desde este marco se pensó la ejecución de la propuesta investigativa "Reconstrucción de la memoria colectiva de los cuidados para la salud" de la población rural del Valle del río Cimitarra".

"El Valle del río Cimitarra es una región inmersa en la región del Magdalena Medio colombiano, en la franja occidental del río Magdalena, entre los departamentos de Antioquia y Bolívar. Se forma en el trayecto que hace este río en búsqueda del Magdalena, y que recorre zonas rurales de los municipios de Remedios y Yondó, en Antioquia; y Cantagallo y San Pablo, en el sur de Bolívar, donde finalmente desemboca (1)".

De reciente colonización, década sesenta y setenta, su población es en su mayoría desplazados forzadamente que entraron a esta reserva forestal en busca de una nueva opción de vida, frente a la embestida paramilitar en regiones como el Urabá, Chocó, Antioquia, Santander y diferentes regiones de la costa Pacífica y Atlántica colombiana.

Sin embargo, en este territorio también se han visto amenazados por el mismo fantasma que los condujo a ella: el desplazamiento forzado, el conflicto social y armado, el abandono del Estado e intereses de los grandes latifundistas del Magdalena Medio y de diversas multinacionales extranjeras que han fijado su mirada en estas ricas tierras. Frente a esto, la decisión de estos campesinos no ha sido la de huir, sino de un proceso organizativo de resistencia campesina que adelantan desde hace veinte años.
Así, la vida en esta región rural se desarrolla entre el temor a la violencia, el fragor de la organización y la participación comunitaria en torno a la sobrevivencia y una apuesta por un futuro mejor.

Sin pretender ser una propuesta acabada, en este artículo se presenta la "ruta" que se propone seguir, así como los aspectos metodológicos que se plantean para lograr el objetivo de comprender los cuidados para la salud de la población rural del Valle del río Cimitarra.

\section{Reconstruir la memoria colectiva de los cuidados para la salud. ¿Para qué?}

La historia de un pueblo abarca un sinnúmero de eventos que lo marcan como un tatuaje, permanente e imposible de borrar; y que a la vez define su presente y futuro. Dentro de éstos se encuentran en un lugar privilegiado, pero no aislado del resto, las formas de cuidado de la vida que sellan la continuidad y supervivencia de ésta. Así, quizás más que hablar que las acciones y prácticas de cuidado son parte de la historia, y por tanto de la memoria colectiva, se puede afirmar que en sí es la historia de un pueblo.

Cuidar, cuidarse y cuidar al otro o a los otros son eventos ligados a cada suceso de la historia de un pueblo, y no pueden verse aislados como lo refiere una de las grandes historiadoras de la Enfermería. Parafraseando a Colliere (2), explica que desde el comienzo de la vida, los cuidados existían ya que era necesario "ocuparse" de la vida para que esta pudiera persistir. Cuidar es un acto de vida que tiene como objeto, en primer lugar y por encima de todo, permitir que la vida continúe y se desarrolle, y de ese modo, luchar contra la muerte: la del individuo y la del grupo. Así, los cuidados no se concibieron nunca como un oficio o una profesión, sino como diversas prácticas cotidianas que pretendían asegurar el cuidado de la vida, mediante el manteni- 
miento de las funciones vitales; $y$ asegurar la supervivencia del individuo y del grupo. Así, era necesario cuidar de la mujer de parto, de los niños, de los vivos, pero también de los muertos (2).

Puntualiza Colliere que “...cuidar es, por tanto, mantener la vida asegurando la satisfacción de un conjunto de necesidades indispensables para la vida, pero que son diversos en sus manifestación (2)". Por lo anterior, cuidar se convierte en un acto de vida que ha dado lugar a prácticas cotidianas y diversas, pero a la vez específicas según las necesidades para perpetuar no sólo la vida del individuo, sino la del colectivo. Estas prácticas de cuidado cotidianas que van desde las de alimentación, vestido, vivienda, sexuales, entre muchas otras, a su vez, forjan formas de hacer y costumbres, y se erigen en un patrimonio de ritos y creencias de un grupo.

El cuidado de la vida toma significados según el contexto y las condiciones de cada momento y a la vez, es definido por estos factores. Es así como se delimitan los sistemas de salud que determinan tanto el sustento filosófico de cómo se conciben los conceptos de vida, muerte, salud y enfermedad, como las prácticas de cuidado para cada uno de ellos. También se forjan los "cuidadores" o agentes tradicionales, los materiales y elementos para cuidar, y se precisa la inversión que se hace para el cuidado.

Ahora bien, ¿para qué reconstruir la memoria colectiva de los cuidados para la salud de un pueblo? Se podría empezar por mencionar, tomando a Leininger como referencia, el aporte que este conocimiento puede hacer para la construcción del cúmulo de conocimientos para la Enfermería, disciplina aún joven y en crecimiento (3). También por la imperiosa necesidad, en un país multicultural como lo es Colombia, de acercarse, comprender y respetar la "mirada del otro", del no profesional acerca de cómo se cuida y como éste se sustenta en una "visión" particular del mundo diferente a la del sistema biomédico o hegemónico. Lo anterior, para un quehacer práctico que refleje un cuidado enfermero beneficioso y congruente con los acervos culturales y las condiciones del contexto ambiental. Y, por supuesto, una atención sanitaria del sistema de salud que evidencie el componente cultural enmarcado en las políticas públicas de salud.

Sin embargo, más allá de lo anterior, pensar la reconstrucción de la memoria colectiva de los cuidados para la salud de un grupo humano tiene otras repercusiones.

La memoria como objeto de la historia, es decir, como una perspectiva desde la cual también es posible reconstruir el pasado, es una forma de distinguir y vincular el pasado en relación al presente y al futuro. Así, esta es la herramienta con la cual la sociedad se representa los materiales, a veces fructíferos, a veces estériles, que el pasado aporta para construir el futuro. Esto quiere decir que al hacer la historia de la memoria que se tiene en un determinado presente sobre un determinado pasado, no sólo se puede comprender y conocer en qué consiste el recuerdo sobre un acontecimiento o proceso en particular, sino que también se puede comprender cómo la sociedad que recuerda organiza su presente y planifica su futuro. Es a partir de la memoria que las sociedades sacan lecciones del pasado y de acuerdo a éstas se puede justificar su repetición o su rechazo, ya sea completo o parcial, a la hora de transformar el presente para construir cierto futuro anhelado (4).

Ahora bien, para abordar la memoria colectiva como una constante revisión de pasado a partir de determinadas condiciones económicas, políticas y sociales en el presente, habría que estudiar cómo el pasado $y$ el presente se relacionan y afectan entre sí. El pasado y el presente tienen una relación dialéctica; pensamos "con el pasado", los pueblos se piensan "acerca del pasado". Esta constituye un paso importante en la consolidación de la identidad. La identidad de un pueblo es relevante en la medida que 
da contenido al discurso de la diferencia cultural y permite asumir la realidad, desde la experiencia del pasado, hacia un futuro en el cual el poder de las decisiones sea tomado por el mismo pueblo.

Por esto, la memoria colectiva, como la capacidad de individuos y pueblos de actualizar y de maniobrar con el pasado a partir de sus luchas cotidianas, define en cierta medida la posibilidad de alcanzar un empoderamiento real y no impuesto por otros. Proceso de empoderamiento necesario para las comunidades rurales del Valle del río Cimitarra para analizar críticamente sus condiciones objetivas y subjetivas; y tomar decisiones frente a los problemas de salud generados por el abandono estatal, la ausencia de políticas sociales y el conflicto social y armado.

Dentro de esta perspectiva, la construcción de la memoria colectiva ha sido considerada como una constante revisión y recomposición del pasado mediada por las experiencias del presente, proceso en que las categorizaciones e identidades hegemónicas entran en constante revisión, recomposición y negociación (5).

\section{La ruta y la acción investigativa: entretejiendo saberes y quehaceres}

Ahora bien, la pregunta es ¿cómo abordar tan complejo fenómeno? Pensar en una propuesta de tal magnitud se convirtió en un reto que superar la sombra de la simplicidad. Por esto, tres problemas se presentaron: ¿qué temas abordar que permitieran acercarse de la forma más completa posible a la reconstrucción de la memoria colectiva de los cuidados para la salud?, ¿cómo hacerlo, metodológicamente hablando, para comprender y responder de forma completa los objetivos planteados? Finalmente, ¿qué sustentos teóricos asumir para una comprensión de los resultados?

Frente a la propuesta de la Antropología médica, que estudia el eje salud-enfermedadatención, se proponen cinco fases o temas a estudiar que reflejen el quehacer disciplinar, retomando la experiencia investigativa acumulada por el profesor Daniel Eslava ${ }^{1}$. Así, la "ruta", como se ha querido llamar, se describe a continuación.

Construcción de las conceptualizaciones de salud y enfermedad. Enfermar y sanar son parte de las construcciones que hacen los pueblos; y se pudiera entender como complejos conceptos que se construyen y reconstruyen dialécticamente desde la realidad vivida y la interpretación que sobre éstas hacen los individuos dentro de un contexto colectivo. En el caso de la población de esta zona rural, el primer aporte investigativo de esta propuesta evidenció cómo estos dos conceptos se han edificado sobre la base de las condiciones sociopolíticas, económicas y ambientales. En ese sentido, la salud y la enfermedad no fueron definidas como un proceso sino como categorías contrarias de "intranquilidad versus bienestar físico y emocional" determinados por la historia de violencia política, el conflicto social y armado, la colonización de la selva, el abandono estatal y el proceso organizativo campesino; siendo este último elemento que define la salud como un anhelo por el cual sus habitantes luchan y le apuestan a la construcción de un futuro en el cual puedan gozar del bienestar físico y emocional.

Descripción de las prácticas de cuidado. En el contexto particular del Valle del río Cimitarra, las acciones de cuidado de la salud han sido determinadas por las condiciones propias de esta región rural. Así, las actividades para cuidar durante la enfermedad o en el nacimiento de una nueva vida, además de encontrarse llenas de sabiduría e historia, se enmarcan en la cotidianidad, la informa-

${ }^{1}$ Daniel Eslava Albarracín es doctor en Enfermería y director Departamento de Salud de Colectivos de la Pontificia Universidad Javeriana (Bogotá-Colombia). Ha realizado varias investigaciones en estos temas y es asesor del presente trabajo. 
lidad de la sabiduría popular y los recursos que brinda la naturaleza. Sin embargo, dada la situación, en lo cotidiano del cuidado se insertan acciones que han surgido del fragor de los procesos organizativos como el mejoramiento de sus condiciones materiales de vida en el marco del abandono estatal y una lucha constante por el reclamo del respeto a la vida y de vivir ésta con dignidad a todos los actores del conflicto social y armado.

Descripción de los agentes tradicionales de salud de las comunidades. ¿Quienes y como son? ¿Cuáles son sus métodos para cuidar? La diversidad y especificidad de su quehacer son parte de la necesidad de este pueblo, siendo los encargados de cuidar en la enfermedad, pero también en eventos no morbosos como el nacimiento de nuevas vidas. Estos "cuidadores informales o no profesionales" reflejan la visión particular del mundo y consolidan en sus comunidades las diversas prácticas de cuidado.

Descripción de los recursos tradicionales utilizados para el cuidado de la salud por las comunidades. Los recursos que se utilizan para el cuidado de la salud, tanto materiales como los artefactos culturales, sustancias, plantas otros, así como los de tipo espirituales como la invocación y la utilización de la ayuda divina.

Descripción del gasto en salud de las comunidades. Se centra en la inversión que hacen los individuos y las comunidades para el cuidado de su salud, expresado tanto en los recursos monetarios como en los de intercambio, de trabajo; y el tiempo y personas destinados para atender los problemas y cuidados de la salud.

Ahora bien, la segunda inquietud, ¿cómo hacerlo? Frente a la complejidad del objeto de estudio y la experiencia de la investigación en el campo sanitario desde la "perspectiva crítica", y en especial, el movimiento de los estudios socioculturales, se propone la investigación cualitativa. Esta tiene como principal objetivo expandir, comprender o reconstruir la realidad, tal y como la observan los actores de un sistema social previamente definido. Hernández refiere que la investigación cualitativa da profundidad a los datos, de la riqueza interpretativa, la contextualización del ambiente o entorno y aporta un punto de vista natural de los fenómenos así como flexibilidad (6). Así, se busca conceptualizar la realidad con base en el comportamiento de las personas estudiadas, explorando sistemáticamente los conocimientos y valores que comparten los individuos en un determinado contexto espacial y temporal (7).

Esta misma complejidad esboza otro reto dentro de la investigación cualitativa: las técnicas de recolección de la información y los sustentos teóricos para la comprensión fiel de los resultados. Por lo anterior, se traza un intento de triangulación metodológica de estos dos aspectos, entendida como la utilización de distintos métodos y técnicas para la comprensión de un mismo fenómeno de estudio. Así, se privilegiaron las técnicas de las tradiciones de la investigación-acción y la etnografía. Las primeras, con el objetivo de hacer presente la voz de los participantes en la reconstrucción de su propia memoria colectiva y la segunda, para lograr un acercamiento al contexto en el cual se insertan los sujetos de estudio.

La discusión de grupo. Al poner cara a cara a los participantes en la reunión, éstos reconstruyen discursivamente el grupo social al que pertenecen y sus particulares normas de referencia; siendo lo determinante la circulación del discurso del objeto social de la investigación. Discursos que adquieren su máximo valor como representación simbólica del grupo frente al fenómeno social estudiado, y por lo tanto expresan la norma ideal del grupo ante el fenómeno (8).

El dibujo y soportes gráficos. Según Araya (9), el dibujo es una forma de expresión discursiva individual, que requiere para su elaboración una reflexión interior y, por consiguiente, una elaboración más propia y auténtica que "representa" su pensamiento individual forjado en lo colectivo. Esta técnica interrogativa tiene como finalidad poner en evidencia elementos constitutivos y de signi- 
ficación del tema de estudio.

Entrevistas semi estructuradas individuales a profundidad. "Técnica de reiterados encuentros cara a cara con la investigadora/or y las/os participantes. Dichos encuentros están dirigidos hacia la comprensión de las perspectivas que tienen respecto de sus vidas, experiencias o situaciones, tal como se expresan con sus propias palabras"(9).

Observación participante. Es la integración del investigador en el espacio de la comunidad observada, definida por la interacción entre observador y observado en el espacio y tiempo de la comunidad (8).

También, para una triangulación teórica se toman diferentes referentes con el fin de evitar una mirada reducida en el análisis y comprensión del fenómeno de estudio.

Teoría de la universalidad y diversidad de los cuidados culturales. Según Leininger (3), su propuesta teórica se centra en la descripción de las prácticas de cuidado culturales, las cuales pueden ser universales o diversas entre los distintos grupos; y que a la vez, éstas pueden diferir del cuidado enfermero. Su fin es conocer los cuidados culturales: su explicación y génesis; tomar decisiones en la intervención profesional que puede ser de preservación, negociación o reestructuración de los patrones del cuidado cultural. De esta forma se espera brindar un cuidado enfermero coherentemente cultural y benéfico, encaminado a evitar los choques culturales y las actitudes etnocéntricas por parte de los profesionales.

La Sociología médica. Plantea De Souza (10) que el fenómeno salud-enfermedad no puede ser pensado como categorías ahistóricas, sino como un proceso fundamentado en la base material de su producción y con las características biológicas y culturales con las que se manifiesta tanto individual como colectivamente. Esto significa que éste debe ser colocado en relación con la totalidad social y con cada una de sus instancias dentro de la especificidad histórica de su manifestación. Así, el fenómeno salud-enfermedad debe ser asumido como una expresión social e individual y como expresión de contradicciones sociales.

La Antropología médica crítica (AMC). Se basa en los supuestos de que todo el conocimiento relacionado con el cuerpo, la salud y la enfermedad son culturalmente construidos, negociados y renegociados en un proceso dinámico a través del tiempo y el espacio (11). Agrega Fajreldin (12) que la AMC entiende los problemas en salud dentro del contexto de fuerzas políticas y económicas que las circundan, incluyendo a las fuerzas a escala institucional, nacional y global, que estructuran las relaciones humanas, moldean los comportamientos sociales, condicionan las experiencias colectivas, reordenan las ecologías locales y sitúan los significados culturales. Así, hace conexiones entre el macro-nivel del mundo capitalista y el micro-nivel de las creencias de los pacientes acerca de la enfermedad y de sus experiencias con la misma (12).

Finalmente, para analizar la información, se propone el método interpretativo-hermenéutico, el cual reconoce los fenómenos sociales como resultados y efectos de la actividad creadora tanto inmediata como institucionalizada. Por lo tanto, toma como centro del análisis la práctica social, la acción humana y la considera como resultado de condiciones anteriores, exteriores pero también como praxis. Esto significa que el acto humano que atraviesa el medio social conserva las determinaciones, pero transforma el mundo sobre las condiciones dadas, dando cuenta de la acción transformadora sobre el medio social (10).

Se parte de que los fenómenos de la salud deben entenderse como frutos y manifestaciones del acondicionamiento socio-históricas que se vinculan al acceso a servicios, tradiciones culturales, concepciones dominantes vehiculizadas y la interacción de todo esto. Estos son manifestaciones de la vida material, de las carencias, de los límites sociales y del imaginario colectivo (10). 


\section{AGRADECIMIENTOS}

La autora desea expresar sus agradecimientos al Dr. Daniel Eslava, asesor de la propuesta investigativa que se presenta en el manuscrito. Además, a los hombres y mujeres de la Asociación Campesina del Valle del río Cimitarra, inspiradores del presente trabajo.

\section{REFERENCIAS}

1. Asociación Campesina del Valle del río Cimitarra. La Zona de Reserva Campesina del Valle del río Cimitarra: una iniciativa agraria de paz. Agencia prensa rural 2008. [citado 2007 marzo 10]; Disponible en: http://www.prensarural.org/spip/spip. php?article1098

2. Colliére M F. Promover la vida. Madrid: Interamericana. Ed. McGraw-Hill, 1993. Pp. 9-12.

3. Leininger M. Transcultural Nursing Concepts, Theories, Research \& Practices. $2^{\mathrm{a}}$ ed. New York: McGraw-Hill, Inc. College Costom Series; 1995. Pp. 134-135.

4. Menéndez A, Joignant A. La Caja de Pandora. Santiago: Editorial Planeta/Ariel, 1999. Pp. 186.

5. Aquino S. Cultura, identidad y poder en las representaciones del pasado: El caso de los zapotecos serranos del norte de Oaxaca, México. Estudios Atacameños 2003; 26: 71-80.

6. Hernández L. Diseño de investigación en ciencias de la salud y sus fundamentos epistemológicos. Bogotá: ECOE Ediciones, 2001. Pp. 24.

7. Bonilla E, Rodríguez P. Más allá del dilema de los métodos: la investigación en ciencias sociales. 2a ed. Bogotá: Editorial Norma, 2005. Pp. 85-86.

8. Callejo J. Observación, entrevista y grupo de discusión: el silencio de tres prácticas de investigación. Revista española de Salud Pública. 76 (5): 409-422.

9. Araya S. Las representaciones sociales: Ejes teóricos para su discusión. Cuaderno de ciencias sociales 127. San José: FLACSO; 2002. Pp. 55.

10. De Souza Minayo MC. El desafío del conocimiento cualitativo en salud. Buenos Aires: Lugar Editorial; 1997. Pp. 195.

11. Chamorro A, Tocornal C. Prácticas de salud en las comunidades del Salar de Atacama: Hacia una etnografía médica contemporánea. Estudios Atacameños 2005; 30: 117-134.

12. Fajreldin V. Antropología médica para una Epidemiología con enfoque sociocultural. Elementos para la interdisciplinariedad. Ciencia y Trabajo 2006; 8 (20):95-102. 